\title{
THE POINCARÉ HOMOLOGY SPHERE AND ALMOST-SIMPLE KNOTS IN LENS SPACES
}

\author{
KENNETH L. BAKER
}

(Communicated by Daniel Ruberman)

\begin{abstract}
Hedden defined two knots in each lens space that, through analogies with their knot Floer homology and doubly pointed Heegaard diagrams of genus one, may be viewed as generalizations of the two trefoils in $S^{3}$. Rasmussen showed that when the 'left-handed' one is in the homology class of the dual to a Berge knot of type VII, it admits an L-space homology sphere surgery. In this note we give a simple proof that these L-space homology spheres are always the Poincaré homology sphere.
\end{abstract}

Beginning with the standard genus 1 Heegaard diagram for the lens space $L(p, q)$ with $p>q>0$ where an $\alpha$ curve and a $\beta$ curve intersect $p$ times, there are just two simple isotopies of these curves that produce a genus one Heegaard diagram with $p+2$ intersection points. Figure 1 adds two basepoints to a local view of each of the standard diagram (center) and the two isotoped diagrams (left and right) to form doubly pointed Heegaard diagrams for knots in the lens space. The center knot is the simple knot $K(p, q, q+1)$ in the language and notation of [Ras. (Such a knot is called grid number one in [BGH08.) The knots to the left and right are Hedden's knots $T_{L}$ and $T_{R}$ Hed11. The knots $T_{L}$ and $T_{R}$ are each homologous to $K(p, q, q+1)$ since they each differ from $K(p, q, q+1)$ by a crossing change as demonstrated in Figure 2. Due to their knot Floer homologies being as close to that of the simple knots without actually being so, we refer to these two knots as almost-simple.

Rasmussen shows that exactly when $K(p, q, q+1)$ is the dual to a Berge knot of type VII (i.e. when $q^{2}+q+1 \equiv 0 \bmod p$ ) $T_{L}$ admits an integral Dehn surgery producing an integral homology sphere that is also an L-space [Ras, §5]. He states that he determined the surgered manifolds for these $T_{L}$ in lens spaces as large as $L(39,16)$ to actually be the Poincaré homology sphere $P^{3}$ by checking their fundamental groups. That all these $T_{L}$ that are homologous to duals of Berge knots of type VII indeed do admit Poincaré homology sphere surgeries is then one part of Greene's Conjecture 1.9 Gre]. Here we provide a short proof.

Theorem. If $T_{L}$ is homologous to the dual to a Berge knot of type VII, then it admits an integral surgery producing the Poincaré homology sphere.

Received by the editors April 18, 2012.

2010 Mathematics Subject Classification. Primary 57M27.

This work was partially supported by grant No. 209184 from the Simons Foundation. 

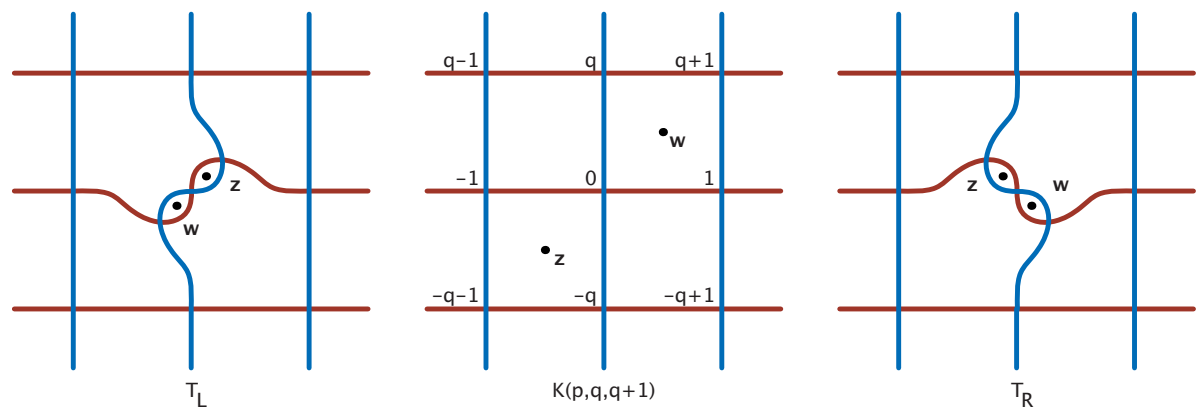

Figure 1

Proof 1. Let $J$ be the positive (left-handed) trefoil, and let $F_{0}$ and $F_{\pi}$ be two of its fibers. Then $F_{0} \cup F_{\pi}$ is a genus 2 Heegaard splitting of $S^{3}$. Now consider an essential simple closed curve in $F_{0}$ as a knot $K$ embedded in $F_{0}$ in $S^{3}$. Then $K$ is immediately a doubly primitive knot ( $[\mathrm{Ber}]$ ) with respect to this Heegaard splitting. The knots in $S^{3}$ that embed as essential curves on the once-punctured torus fiber of the positive trefoil and their mirrors are the Berge knots of type VII Ber.

One may show that the surface framing that $F_{0}$ induces on $K$ has positive integral slope. Dehn surgery along this slope then yields a lens space $L(p, q)$ and the knot $K^{\prime}=K(p, q, q+1)$ dual to $K$. The surgery on $K$ then causes a surgery of $F_{0}$ along $K$, rendering it a disk $D_{0}$ that $K^{\prime}$ intersects twice. Indeed $D_{0} \cup F_{\pi}$ is a Heegaard torus for the lens space $L(p, q)$, and, due to the doubly primitive presentation of $K$, $K^{\prime}$ is an unknotted arc in the solid torus on each side. Notice that in $L(p, q)$, after surgery on $K$, the positive trefoil $J$ is now the boundary of this disk: $J=\partial D_{0}$. Therefore +1 surgery on $J$ in $L(p, q)$ simply inserts a full left-handed twist in $K^{\prime}$. We will conclude that this twist transforms $K^{\prime}$ into $T_{L}$, though for now let us call the resulting knot $T^{\prime}$.

The Poincaré homology sphere $P^{3}$ may be obtained as +1 surgery on $J$ in $S^{3}$. Let us first do this surgery, regard $K \subset F_{0}$ as a knot in $P^{3}$, and then do $F_{0}$-framed surgery on $K$. Because we are only swapping the order in which surgery is done on the link $K \cup J \subset S^{3}$, this must also produce the lens space $L(p, q)$ in which the knot dual to $K \subset P^{3}$ is the above knot $T^{\prime}$, i.e. $K^{\prime}$ with that full left-handed twist. In particular, we conclude that $T^{\prime} \subset L(p, q)$ is a 1-bridge knot with respect to the Heegaard torus $D_{0} \cup F_{\pi}$, is in the homology class of a knot dual to a Berge knot of type VII, and admits an integral Poincaré homology sphere surgery.

Because the Poincaré sphere $P^{3}$ is both an L-space and an integral homology sphere, [Ras, Proposition 4.5] implies that since $T^{\prime}$ is a knot in the lens space $L(p, q)$ representing a primitive homology class (that of $K^{\prime}=K(p, q, q+1)$ ) and admitting an integral surgery to $P^{3}$, then $\operatorname{rk}(\widehat{\operatorname{HFK}}(\mathrm{L}(\mathrm{p}, \mathrm{q}), \mathrm{T})) \leq \mathrm{p}+2$. Because $T^{\prime}$ is 1-bridge but generically not the simple knot $K(p, q, q+1)$ (otherwise $T^{\prime}=K(p, q, q+1$ ) is actually dual to the trefoil $J$, the only knot in $S^{3}$ with an integral surgery to $P^{3}$ [Ghi08]), we must have that $T^{\prime}=T_{L}$ by [Hed11, Proposition 3.3].

If one were to pay more attention to the meridional disks for handlebodies to either side of $F_{0} \cup F_{\pi}$ that are disjoint from $K$, one could directly see that the twist on $J$ in $L(p, q)$ takes $K^{\prime}=K(p, q, q+1)$ to $T_{L}$ without appealing to the knot Floer 


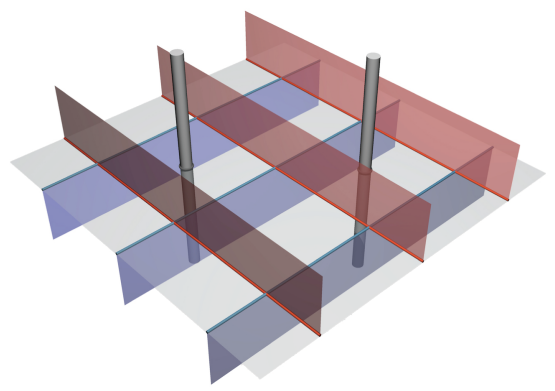

$K(p, q, q+1)$

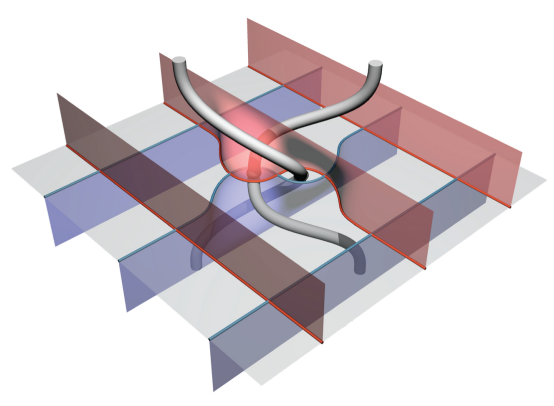

$T_{L}$

FiguRE 2

homology argument in the last paragraph of the proof above. Indeed, $J$ is the loop in the local picture of Heegaard torus in the center of Figure 1 that encircles the two basepoints. Figure 2 then illustrates that $T_{L}$ is obtained from $K(p, q, q+1)$ by a full Dehn twist on this curve.

The manifolds, Heegaard splittings, knots, and fibers in the proof above all behave well with respect to certain involutions. Let us therefore recast that proof in terms of bandings between a two-bridge link $\ell$ and each an unknot $\hat{s}$ and the $(-3,5)$-torus knot $\hat{p}$. We omit details of the correspondences.

Proof 2. Let $A$ be the braid axis of the closure $\hat{s}$ of the 3 -braid $\sigma_{1} \sigma_{2}$. The positive trefoil $J$ may be viewed as the lift to $S^{3}$ of $A$ in the double cover of $S^{3}$ branched

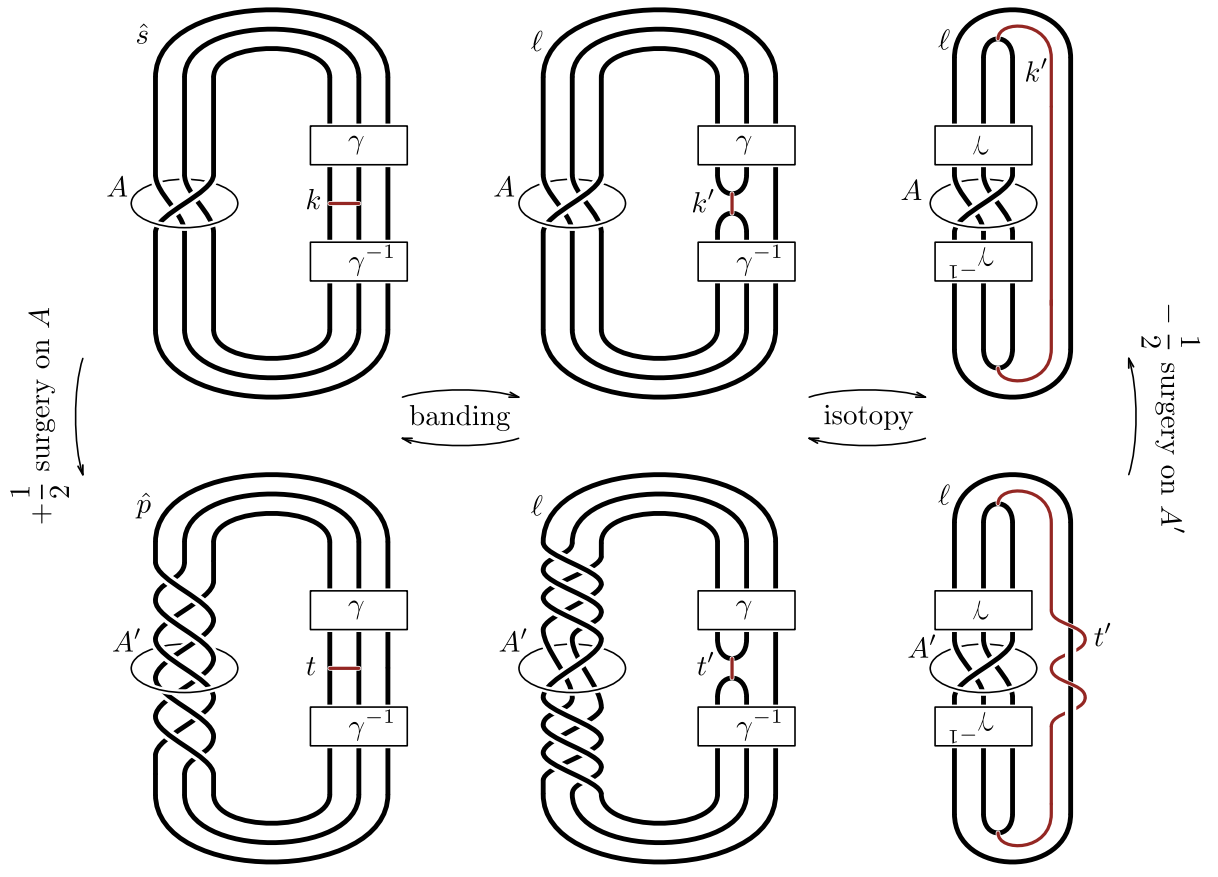

FiguRE 3 
over the unknot $\hat{s}$. A disk $D$ bounded by $A$ that intersects $\hat{s}$ three times lifts to a once-punctured torus fiber $F$ of $J$. Any essential simple closed curve on $F$ is then isotopic on $F$ to a curve that is the lift of some simple arc $k$ on $D$ connecting two of the three points of $D \cap \hat{s}$. We may simplify the presentation of this arc with a 3 -braid conjugation. The top-left of Figure 3 shows this setup where $\gamma$ is the conjugating 3 -braid. Banding together the unknot $\hat{s}$ along $k$ with the framing bestowed upon it by $D$, we obtain a two-bridge link $\ell$ and a dual arc $k^{\prime}$ as shown in the top-middle and top-right of Figure 3. Observe that $A$ bounds a disk that intersects each $\ell$ and $k^{\prime}$ just once.
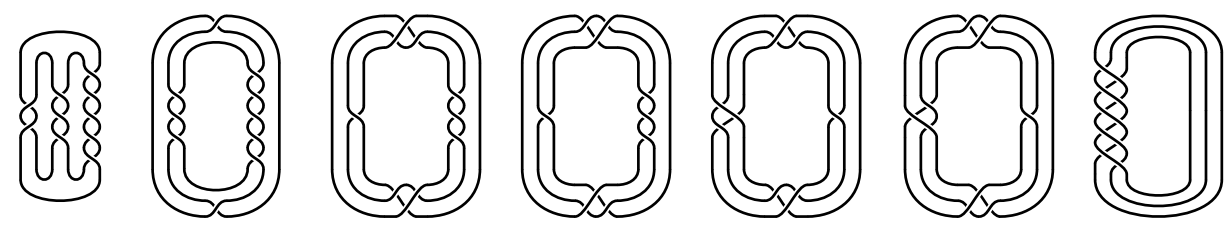

Figure 4

Performing $+1 / 2-$ Dehn surgery along $A$ before the banding takes the closed $3-$ braid $\hat{s}$ to $\hat{p}$, the closure of the 3 -braid $\sigma_{1} \sigma_{2}\left(\sigma_{1} \sigma_{2}\right)^{-6}=\left(\sigma_{1} \sigma_{2}\right)^{-5}$, as in the lower-left of Figure 3 . The closed braid $\hat{p}$ is the $(-3,5)$-torus knot, and its double branched cover is $P^{3}$. (The pretzel knot $P(2,-3,-5)$ is isotopic to the $(-3,5)$-torus knot; see Figure 4.) Doing this surgery on $A$ after the banding does not change $\ell$, but does cause $a^{\prime}$ to wrap around $\ell$ twice near $\ell \cap D$, giving an arc $t^{\prime}$ as in the lower-right of Figure 3. Keeping the framings in mind, a banding of the two-bridge link $\ell$ along this arc $t^{\prime}$ produces the $(-3,5)$-torus knot $\widehat{p}$.

\section{REFERENCES}

[Ber] John Berge, Some knots with surgeries yielding lens spaces, unpublished manuscript.

[BGH08] Kenneth L. Baker, J. Elisenda Grigsby, and Matthew Hedden, Grid diagrams for lens spaces and combinatorial knot Floer homology, Int. Math. Res. Not. IMRN 10 (2008), Art. ID rnm024, 39, DOI 10.1093/imrn/rnn024. MR2429242(2009h:57012)

[Ghi08] Paolo Ghiggini, Knot Floer homology detects genus-one fibred knots, Amer. J. Math. 130 (2008), no. 5, 1151-1169, DOI 10.1353/ajm.0.0016. MR2450204(2010f:57013)

[Gre] Josh Greene, The lens space realization problem, arXiv:1010.6257 [math.GT].

[Hed11] Matthew Hedden, On Floer homology and the Berge conjecture on knots admitting lens space surgeries, Trans. Amer. Math. Soc. 363 (2011), no. 2, 949-968, DOI 10.1090/S0002-9947-2010-05117-7. MR2728591 (2012f:57006)

[Ras] J. Rasmussen, Lens space surgeries and L-space homology spheres, arXiv:0710.2531v1 [math.GT].

Department of Mathematics, University of Miami, P.O. Box 249085, Coral Gables, FLORIDA 33124-4250

E-mail address: k.baker@math.miami.edu

$U R L:$ http://math.miami.edu/kenken 